\title{
Compressive Sensing Reconstruction for EDS Analysis
}

\author{
Joshua A. Taillon ${ }^{1}$ \\ 1. Material Measurement Laboratory, National Institute of Standards and Technology, Gaithersburg, \\ MD, USA
}

Among the many challenges faced by a microanalyst during a scanning/transmission electron microscopy (SEM/TEM) X-ray energy dispersive spectroscopy (EDS) experiment, limiting the radiation dose delivered to the sample is often one of the most important, especially during the analysis of beamsensitive specimens, such as biological and polymeric materials. Reducing electron dose by limiting pixel dwell times can alleviate much of the damage, at the expense of total X-ray generation, and by extension the signal to noise ratio (SNR) and overall quality of the resulting spectroscopic data. This problem is especially pernicious during two- or three-dimensional EDS mapping experiments, where dwell times are often particularly short, and total experimental collection times can run excessively long.

Recent developments in electron microscopy have borrowed a technique from the mathematics community called compressive sensing (CS) [1], which provides a mechanism for correctly inpainting missing data from highly undersampled (i.e. sparse) signals, subject to restrictions on how the signal is collected. This approach was first demonstrated in TEM tomography, reducing the number of tilted images required for a high-quality reconstruction [2]. Following this, CS was proposed for reducing dose in scanning TEM (STEM) imaging [3], and has since been demonstrated for both SEM imaging [4] and hyperspectral imaging via STEM-EELS (electron energy-loss spectroscopy) [5], reducing the total electron dose delivered to the sample. To the author's knowledge, the present report of CS-enhanced EDS analysis is the first to be demonstrated within the literature.

In this study, a CS reconstruction algorithm developed for hyperspectral imagery known as Beta Process Factor Analysis (BPFA) [6] was applied to EDS data collected on an SEM platform. The techniques are expected to be generalizable to STEM, as well as similar spectroscopic techniques, such as X-ray Fluorescence Spectrometry (XRF). In a typical CS reconstruction, the underlying data must be sampled in an incoherent manner (i.e. randomly sampled) to ensure a successful inpainting reconstruction [4]. In this work, a standard raster-based scanning approach was utilized, relying instead on the probabilistic nature of x-ray generation and low dwell times (approximately $50 \mu \mathrm{s}$ ) to provide the sparse (pseudo)random sampling of the hyperspectral datacube.

A two-dimensional EDS map was acquired from an aluminum silicate sample with an electron beam accelerating voltage of $5 \mathrm{kV}$. An example of the collected data, together with the result of the BPFA reconstruction is shown in Figure 1. Spectra from two points within the map are included to demonstrate the sparse nature of the collected data, and the drastically improved signal in the reconstructed result. In the reconstruction, the spectra are fully-featured and have substantially reduced noise.

Figure 2 illustrates the enhancement observed in the line intensity maps after reconstruction using the BPFA algorithm. For the given sample, the SNR of each elemental map is indicated, showing enhancement of the signal ranging from a 17\% improvement (for the Al-K $\mathrm{K}_{\alpha}$ line) to a $396 \%$ improvement (for $\mathrm{N}-\mathrm{K}_{\alpha}$ ). Qualitatively, the interpretability of the maps is also significantly improved. These initial results indicate that BPFA CS reconstruction can successfully improve severely 
undersampled EDS maps, warranting added research in the quantitative effects of such a reconstruction.

References:

[1] EJ Candès, J Romberg, and T Tao, IEEE Transactions on Information Theory, 52 (2006), p. 489.

[2] Z Saghi et al., Nano Letters, 11 (2011), p. 4666.

[3] A Stevens, H Yang, L Carin, I Arslan, and ND Browning, Microscopy, 63 (2014), p. 41.

[4] K Hujsak et al., Microscopy and Microanalysis 22 (2016), p. 778.

[5] A Stevens et al., Microscopy and Microanalysis 22(S3) (2016), p. 560.

[6] Z. Xing et al., SIAM Journal on Imaging Sciences 5 (2012), p. 33.
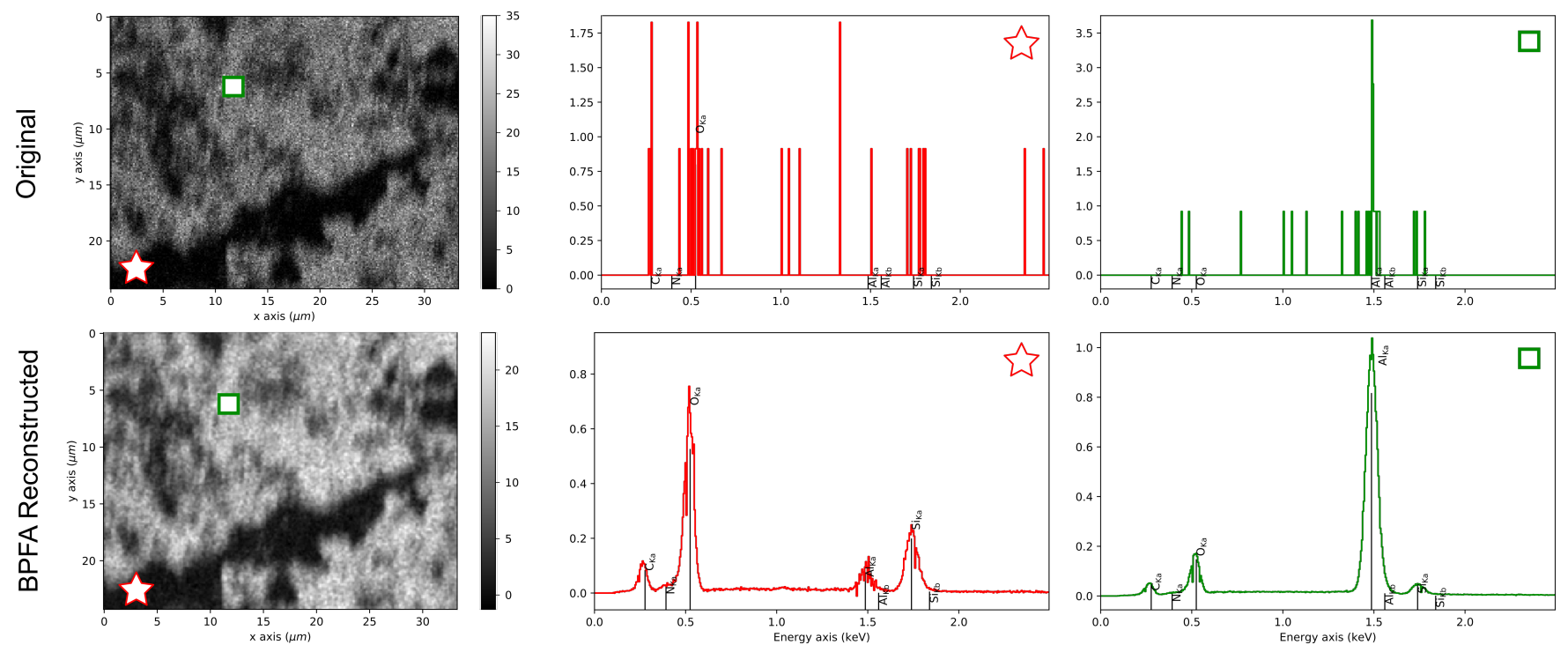

Figure 1. Results of a BPFA compressed sensing reconstruction on an SEM-EDS map of an aluminum silicate sample. The leftmost column shows the Al- $\mathrm{K}_{\alpha}$ intensity map, indicating the locations of the single-pixel spectra shown in the center (star) and right (square) columns. The top row indicates the ascollected data; the bottom shows the data after BPFA reconstruction, demonstrating the substantial improvement in signal quality after reconstruction.
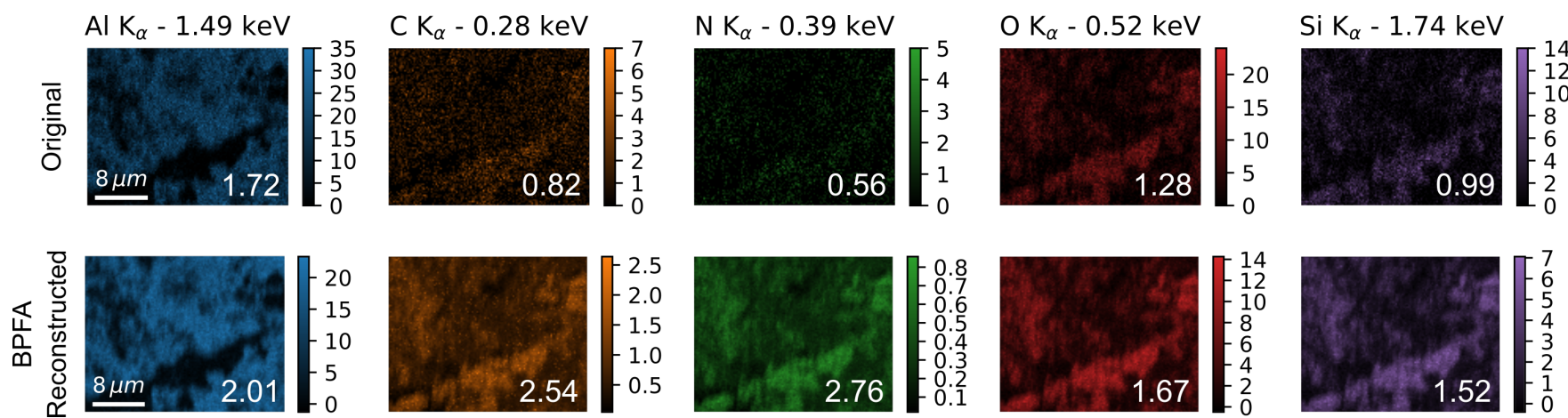

Figure 2. X-ray line intensity maps of the five constituent elements in an EDS map of an aluminum silicate sample. The top row is the original data as-collected, and the bottom row represents the same maps after BPFA reconstruction using the methods of Ref. [6], showing considerable qualitative enhancement in map interpretability, without loss of spatial resolution. The numbers in each image represent the SNR, defined as the ratio of the signal mean to the signal standard deviation $\left(\mu_{\mathrm{sig}} / \sigma_{\mathrm{sig}}\right)$. 